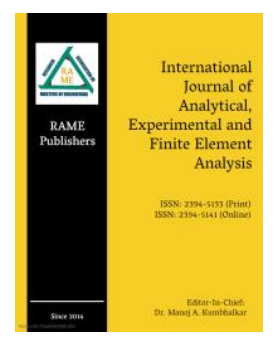

Deven Subhash Darak devendarak77@gmail.com

Prathamesh Prasad Gogate prathameshgogate1612@gmail.com

\section{Saravanakumar Shanmugaiah} saravana061297@gmail.com

Kaif Mukhtar Shaikh kaif87542@gmail.com

Department of Mechanical

Engineering, JSPM Narhe Technical Campus, Pune, India

\title{
Study of Rail-Wheel Performance during Curve Negotiation
}

Abstract - Failure in wheels mainly occurs when the train travels on a curved track. When a train runs on a curve maximum load on one wheel. Three types of forces are applied on the wheel while in contact with the rail. Three-dimensional finite element model of the wheel rail interaction is used to investigate the effect of applied loading forces on the wheels. The Interaction between left and right wheel is considered. The wheels are analyzed for the effect of forces on wheels such as Vertical force, Centrifugal force and also Lateral force. This paper also discusses the comparison of safe speed and radius of curvature. The model has been created in Catia software and the finite element analysis has been done in software code and ANSYS. The proposed simulation accurately shows the real geometry and the wheel rail interface. The numerical results show that the speed and radius of curvature are inversely proportional.

Keywords - Curved track, Finite element method, Rail-wheel, FEA.

\section{INTRODUCTION}

Rail transportation is considered to be the safest one worldwide. Because rail transit is complicated, such tragedies appear to be difficult to entirely eradicate, regardless of the amount of money spent on improving the protection. Due to the constant rise in train speed, further safety is required in rail operations. In general, by using a safety process, the rail sector is always focused on decreasing the possibility of an unwanted Derailment. For example, in the case of breakdowns in mechanical parts that control the wheel on rails or in an aberrant conduct of the driver, these techniques don't always look adequate. Conducting at a pace in speed zones [1-5].

The main mechchanism explains that the resulting force vector, whose components include the centrifuge force,

Research Paper - Peer Reviewed

Published online - 30 June 2021

\section{(C) 2021 RAME Publishers}

This is an open access article under the CC BY 4.0 International License https://creativecommons.org/licenses/by/4.0/

Cite this article - Deven Subhash Darak, Prathamesh Prasad Gogate, Saravanakumar Shanmugaiah, Kaif Mukhtar Shaikh, "Study of RailWheel performance during Curve Negotiation", International Journal of Analytical, Experimental and Finite Element Analysis, RAME Publishers, vol. 8 , issue 2 , pp. 48-53, 2021

https://doi.org/10.26706/ijaefea.2.8.20210607 gravity and inertia force of the lateral and vertical vibrations, can be driven from the gravity c outside of the external (high-rail) rail when operation is much faster than the speed-limit set by the curved track. [1, 6-8]

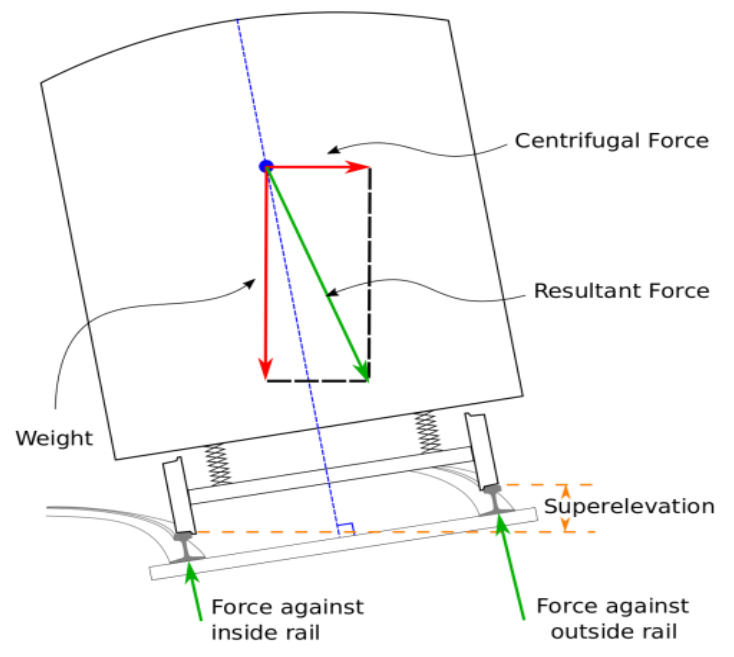

Figure 1. Forces acting on wheel [3-5]

In this paper we have considered a wheel set of WAG9 and the weight of the engine has been divided with the number of axles. A FEM has been used for analysis of curved track and wheel. A three dimensional model has been created and analysis of the model is done by applying three different type of forces such as Vertical force, Lateral force and Longitudinal force. 


\section{VERTICAL FORCE}

These are the reaction forces that act on the wheel in the vertical direction. Reaction force is just a reaction developed when we apply a force. This force acts in $\mathrm{X}$ direction. These reaction forces vary with speed. We need to consider the axle as a beam and the reaction forces are acted on the wheel. There are various parameters which affect the design and maintenance of horizontal curves are radius (R), cant/superelevation (C), equilibrium speed $\left(\mathrm{V}_{\mathrm{eq}}\right)$, cant deficiency $\left(\mathrm{C}_{\mathrm{d}}\right)$, cant excess $\left(\mathrm{C}_{\mathrm{ex}}\right)$ and cant angle. The dimensions to analyse the lateral effect on the track over broad gauge (BG) track [9-14]. Some of the considerations are shown in the table below which will help us to calculate resultant forces.

TABLE 1

BASIC CONSIDERATIONS FOR SOLVING FORCES.

\begin{tabular}{|c|c|c|c|c|}
\hline $\begin{array}{l}\text { Chord } \\
\text { angle }\end{array}$ & $\begin{array}{l}\text { Curve } \\
\text { radius } \\
(\mathrm{m})\end{array}$ & $\begin{array}{c}\text { Safe speed } \\
(\mathrm{km} / \mathrm{hr})\end{array}$ & $\begin{array}{c}\text { Cant or Super } \\
\text { elevation }\end{array}$ & $\begin{array}{l}\text { Cant } \\
\text { angle }\end{array}$ \\
\hline $1^{\circ}$ & 1750 & 180.34 & 256.08 & 8.68 \\
\hline $2^{\circ}$ & 875 & 124.83 & 245.39 & 8.32 \\
\hline $3^{\circ}$ & 583.34 & 99.69 & 234.75 & 7.97 \\
\hline $4^{\circ}$ & 437.5 & 84.34 & 224 & 7.61 \\
\hline $5^{\circ}$ & 350 & 73.62 & 213.38 & 7.25 \\
\hline $6^{\circ}$ & 291.67 & 65.50 & 202.69 & 6.89 \\
\hline $7^{\circ}$ & 250 & 59.03 & 192.06 & 6.53 \\
\hline $8^{\circ}$ & 218.75 & 53.66 & 181.37 & 6.17 \\
\hline $9^{\circ}$ & 194.45 & 49.08 & 170.07 & 5.81 \\
\hline $10^{\circ}$ & 175 & 45.08 & 160 & 5.45 \\
\hline
\end{tabular}

By using the table 1, two reaction forces can be calculated. One reaction is on the inner rail and another reaction is on the outside rail. The below diagram shows distribution of forces [3-5].

Formula for finding resultant forces,

$$
\begin{aligned}
\mathrm{R}_{\mathrm{wA}}=\mathrm{R}_{\mathrm{a}}= & \left(\frac{m V^{2}}{R} \sin \theta+m g \cos \theta\right) \frac{1}{2} \\
& +\left(m g \sin \theta-\frac{m V^{2}}{R} \cos \theta\right) \frac{h}{x}
\end{aligned}
$$

Similarly, we can find $\mathrm{R}_{\mathrm{wB}}$

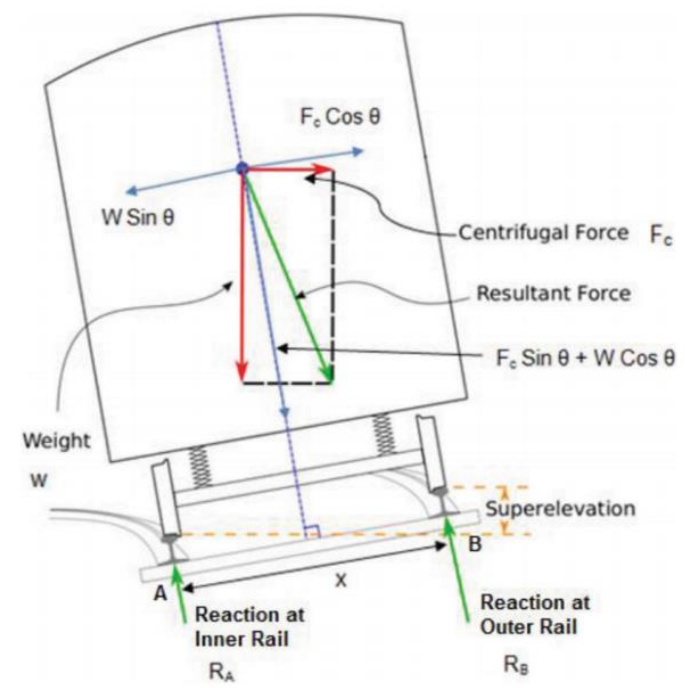

Figure 2. Distribution of forces [3-5]

Gyroscopic couple,

$$
\mathrm{C}=\mathrm{I} \omega_{\mathrm{w}} \cos \theta \omega_{\mathrm{p}}
$$

Reaction at gyroscopic couple,

$$
\mathrm{R}_{\mathrm{GA}}=\mathrm{R}_{\mathrm{GB}}=\mathrm{CG} / \mathrm{x}
$$

Reaction at outer rail,

$$
\mathrm{R}_{\mathrm{B}}=\uparrow \mathrm{R}_{\mathrm{wB}}+\uparrow \mathrm{R}_{\mathrm{GB}}
$$

Reaction at inner rail,

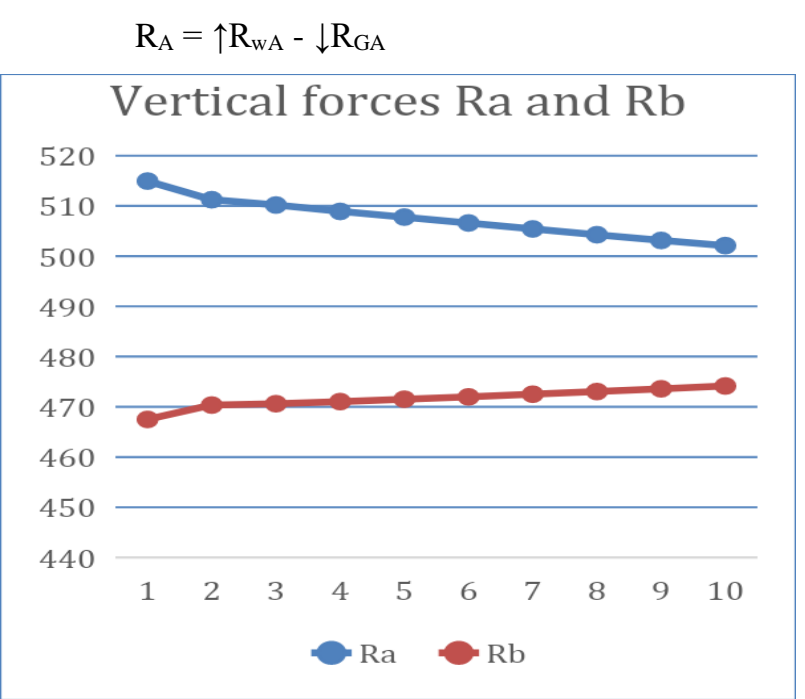

Figure 3. Graph of reaction forces

\section{LATERAL FORCE}

The force acting in the direction parallel to the ground and perpendicular to the direction of gravity traction of the earth is called lateral forces. It is different from longitudinal force in such a way that in case of lateral force, the path of the force is parallel to the plane of reference of structure and perpendicular to the axis of 
rotation of the structure. Lateral force is the centrifugal force that act away from the wheel. This force always acts in $\mathrm{Y}$ direction [15-19]. The formula of Centrifugal force is $\mathrm{F}_{\mathrm{c}}=\frac{m V^{2}}{R}$. The speed taken is safe speed for accurate results.

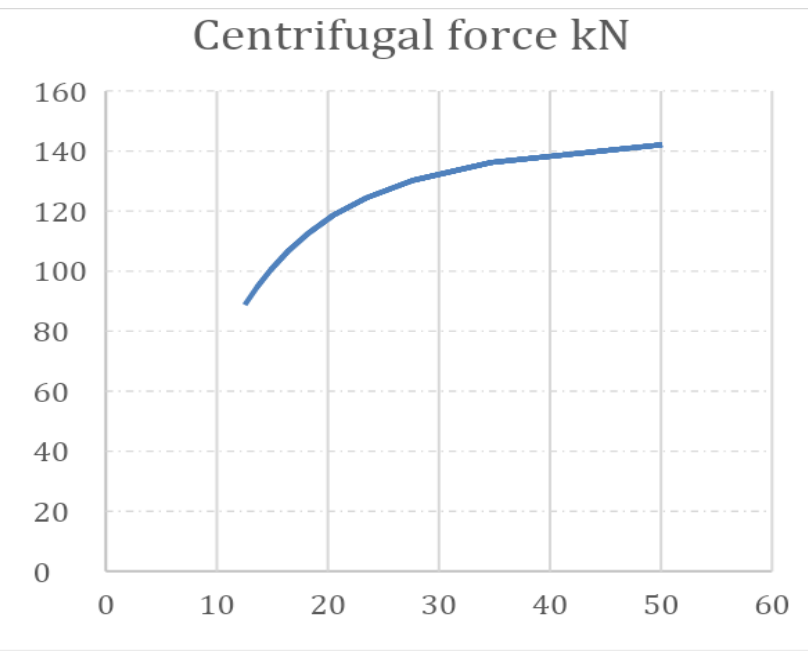

Figure 4. Safe speed vs Centrifugal force

\section{LONGITUDINAL FORCE}

Longitudinal force always acts in the $\mathrm{Z}$ direction. In Rail wheel condition the Longitudinal force is the Tractive effort or Tractive force. Tractive force is the force used to generate motion between a body and a tangential surface. Tractive force is used to describe the pulling or pushing capability of a locomotive Values for traction force vary with weight of the bogie [20-23]. As we have taken WAG9 the value for the tractive force can be observed by the below figure 5 .

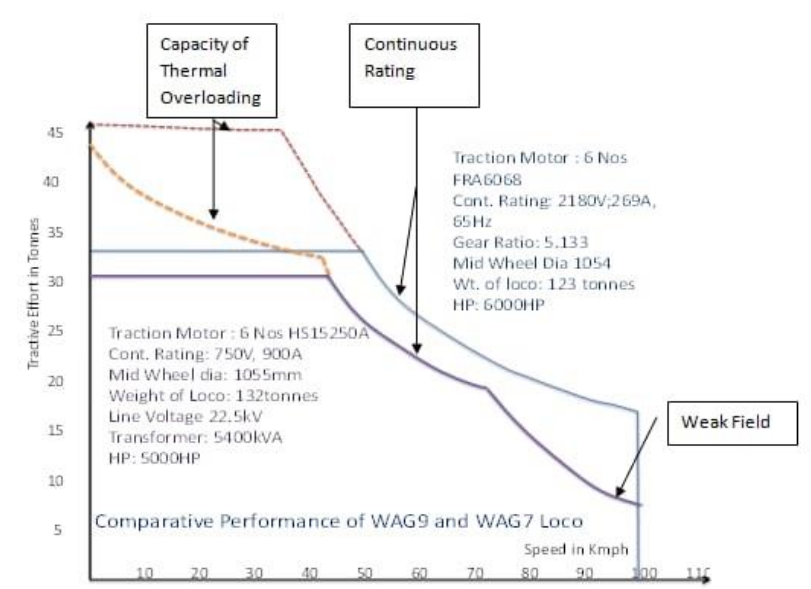

Figure 5. Comparison of tractive force between WAG9 and WAG7 Loco [2]
TABLE II

TRACTIVE FORCE ON EACH AXLE

\begin{tabular}{|c|c|c|}
\hline $\begin{array}{c}\text { Safe speed } \\
(\mathrm{km} / \mathrm{hr})\end{array}$ & $\begin{array}{c}\text { Total tractive } \\
\text { effort } \\
(\mathrm{kN})\end{array}$ & $\begin{array}{c}\text { Tractive effort on each } \\
\text { axle } \\
(\mathrm{kN})\end{array}$ \\
\hline 180.34 & Negligible & Negligible \\
\hline 124.83 & Negligible & Negligible \\
\hline 99.69 & Negligible & Negligible \\
\hline 84.34 & 196.13 & 32.68 \\
\hline 73.62 & 215.75 & 36 \\
\hline 65.5 & 245.17 & 40.18 \\
\hline 59.03 & 254.97 & 42.49 \\
\hline 53.66 & 313.81 & 52.30 \\
\hline 49.08 & 333.43 & 55.57 \\
\hline 45.08 & 333.43 & 55.57 \\
\hline
\end{tabular}

From table 2, it can be seen that tractive force decreases with speed. Once the speed passes $100 \mathrm{~km} / \mathrm{hr}$ the tractive force becomes negligible.

\section{Finite ELEMENT ANALYSIS ON RAILWAY WHEEL}

Finite element method is used to analyses the response of the rail joint to the static and dynamic load. The Finite Element analysis is performed using structural analysis of the ANSYS R19 workbench software after imported 3D assemblies form CATIA software. ANSYS is a generalpurpose finite element of modeling package for numerical solved problems. Like any finite element software, ANSYS solves governing differential equations by breaking the problem into small elements. When the wheel is contact element and the rail is target element, the wheel and rail will be different.

Through upgrading existing ways to analyze the impact of wheel motion on the rail, finite element analysis (FEA) is used as a tool for contact mechanics modelling, assessment, and simulation of the Rail-Wheel contact. The majority of traditional methods and computational approaches are limited to static solutions that do not account for the whole rolling of the wheel. Furthermore, various railway system model simplifications, such as disregarding the setting pads in these investigations, reduced the precision of the result. 


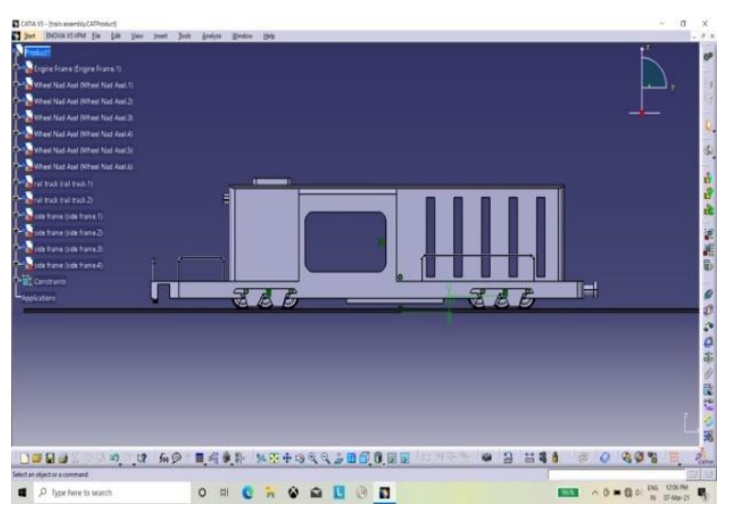

Figure 4: CAD model of WAG9 engine

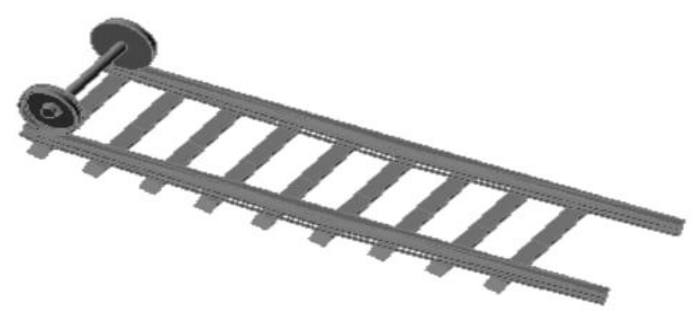

Figure 5: CAD model of curved track

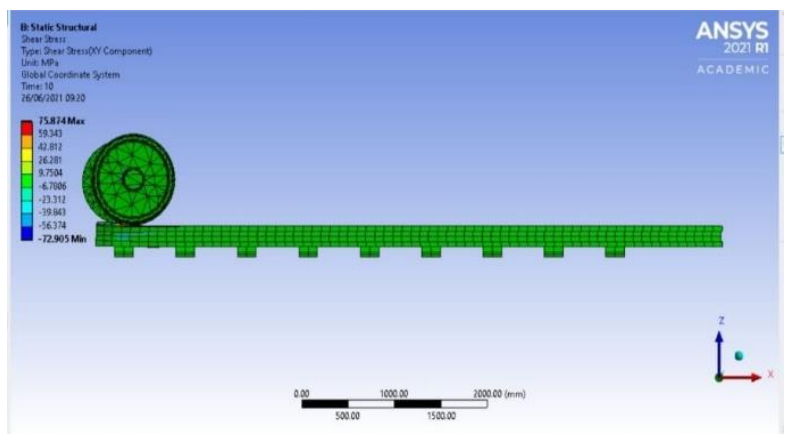

Figure 6: Shear stress on model

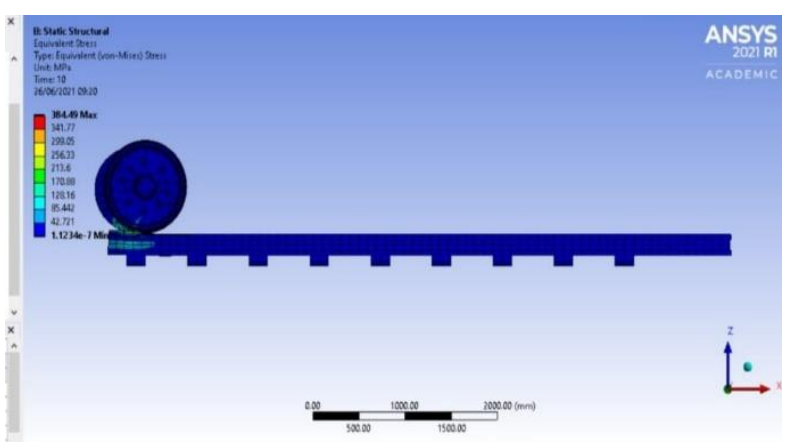

Figure 7: Equivalent stress on model

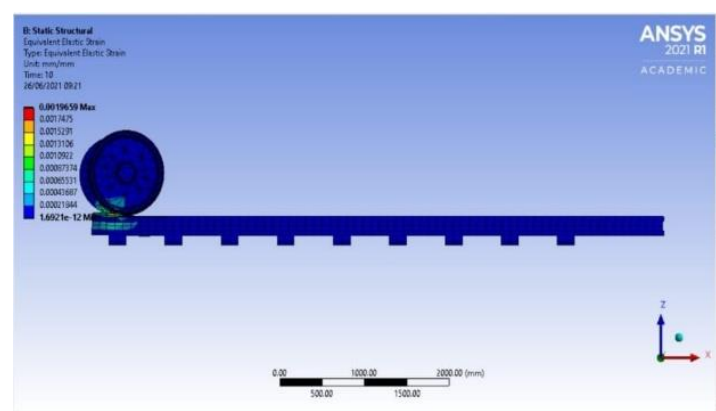

Figure 8: Equivalent elastic strain on model

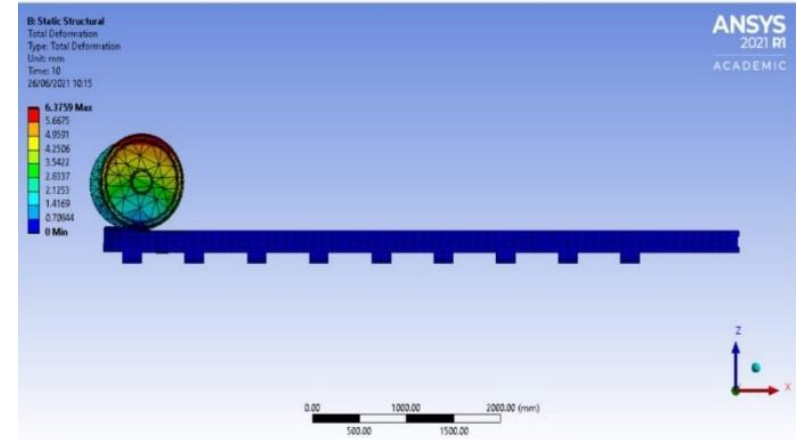

Figure 9: Total deformation of model

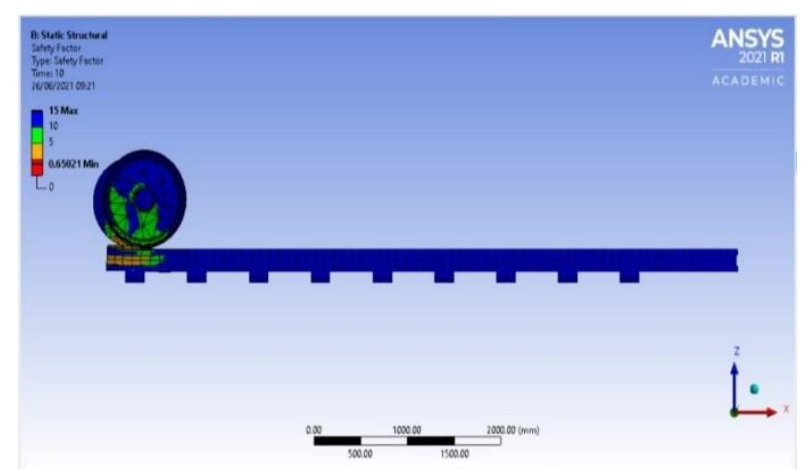

Figure 10: Factor of safety of model

\section{RESULTS FROM FEA}

Below are some of the graphs of Shear stress, Equivalent stress and Total deformation to the Chord angle. It can be seen that all the factors increase with increase in chord angle.

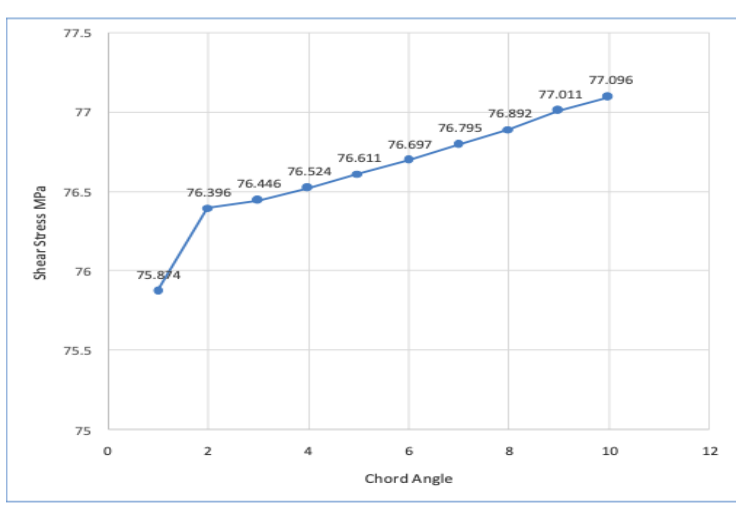

Figure 11. Shear Stress (MPa) vs Chord Angle

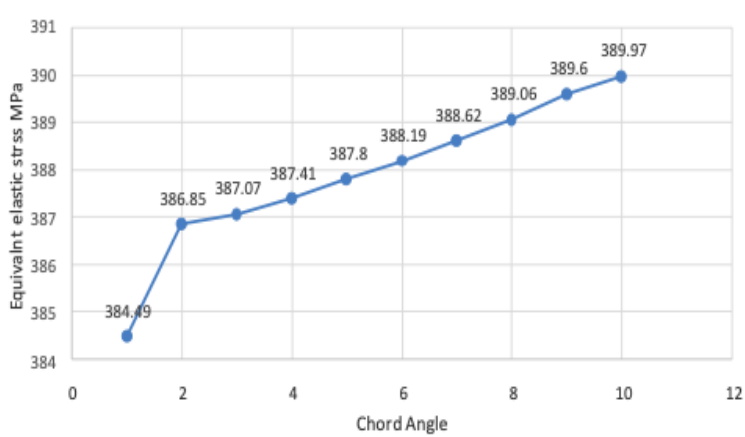

Figure 12. Chord angle vs Equivalent elastic strain 


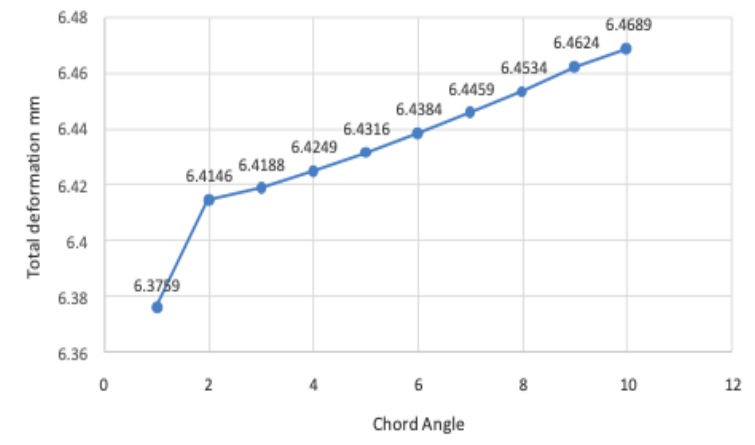

Figure 13. Chord angle vs Total deformation

\section{CONCLUSION}

In this study, track vertical stiffness has been detected from experimental results of track settlements using a FEM model. Before investigating the vehicle-track system dynamics in curves and the derailment risk, a new technique for modelling the fastener as a clip with a compressive force determined from the tightening torque has been described. It can be concluded based on the findings that:

1. Fastener failure causes lateral forces, which are proportional to uncompensated acceleration regardless of whether the vehicle negotiates the curve with cant excess or deficiency. Vertical forces are not importantly influenced by the fasteners' failure.

2. The vertical displacement applied to the shaft is dependent on the magnitude of vertical forces, which has been established by previous research using both experimental and numerical analysis.

3. As we checked for all 10 cases: -

Case1. The maximum Equivalent stress is 384.49 $\mathrm{MPa}$, maximum Shear Stress is $75.874 \mathrm{MPa}$, Total Deformation is $6.3759 \mathrm{~mm}$, Equivalent Elastic Strain is 0.0019659 . Factor of Safety is 15 .

Case 10. The maximum equivalent stress is 389.97 $\mathrm{MPa}$, maximum Shear Stress is $77.096 \mathrm{MPa}$, Total Deformation is $6.4689 \mathrm{~mm}$, Equivalent Elastic Strain is 0.00197 . Factor of Safety is 15.

- Total deformation $(\max )$ increases steadily in all the 10 cases.

- Values of equivalent elastic strain are almost similar in all the cases.
- The Value of equivalent stress (max) is very high in case 10.

We determined Equivalent stress and compared it to the ultimate tensile strength of steel, and based on the results, we can conclude that the wheel will not fail under the present conditions.

\section{REFERENCES}

[1] J. Xu, P. Wang, Y. Gao, J. Chen, and R. Chen, "Geometry evolution of rail weld irregularity and the effect on wheelrail dynamic interaction in heavy haul railways," Eng. Fail. Anal., vol. 81, pp. 31-44, 2017.

[2] Mahesh Kumar Jain, "Tractive Effort", May 122013. Available at https://www.railelectrica.com/tractionmechanics/tractive-effort/

[3] M. A. Kumbhalkar, D. V. Bhope, and A. V. Vanalkar, "Analysis of Rail Vehicle Suspension Spring with Special Emphasis on Curving, Tracking and Tractive Efforts," IOP Conf. Ser. Mater. Sci. Eng., vol. 149, no. 1, 2016.

[4] M A Kumbhalkar, D V Bhope, A V Vanalkar, P P Chaoji, "Failure analysis of primary suspension spring of rail road vehicle," Journal of failure analysis and prevention, vol. 18, no. 6, 2018.

[5] M A Kumbhalkar, D V Bhope, A V Vanalkar, P P Chaoji, "Investigation for Failure Response of Suspension Spring of Railway Vehicle: A Categorical Literature Review," Journal of failure analysis and prevention, vol. 20, no. 4, 2020.

[6] Y. Hu et al., "Investigation on wear and rolling contact fatigue of wheel-rail materials under various wheel/rail hardness ratio and creepage conditions," Tribol. Int., vol. 143, p. 106091, 2020.

[7] Y. B. Huang, L. B. Shi, X. J. Zhao, Z. B. Cai, Q. Y. Liu, and W. J. Wang, "On the formation and damage mechanism of rolling contact fatigue surface cracks of wheel/rail under the dry condition," Wear, vol. 400-401, pp. 62-73, 2018.

[8] X. J. Zhao et al., "Effect of spherical dents on microstructure evolution and rolling contact fatigue of wheel/rail materials," Tribol. Int., vol. 127, pp. 520-532, 2018.

[9] X. Cao, L. B. Shi, Z. B. Cai, Q. Y. Liu, Z. R. Zhou, and W. J. Wang, "Investigation on the microstructure and damage characteristics of wheel and rail materials subject to laser dispersed quenching," Appl. Surf. Sci., vol. 450, pp. 468483, 2018.

[10] M A Kumbhalkar, D V Bhope, A V Vanalkar, "Finite element analysis of rail vehicle suspension spring for its fatigue life improvement," Advanced manufacturing and 
materials science, Lecture Notes on Multidisciplinary Industrial Engineering, 2018.

[11] L. Prates Ferreira de Almeida, L. Entringer Falqueto, H. Goldenstein, A. Cesar Bozzi, and C. Scandian, "Study of sliding wear of the wheel flange - Rail gauge corner contact conditions: Comparative between cast and forged steel wheel materials," Wear, vol. 432-433, 2019.

[12] A. Shebani and S. Iwnicki, "Prediction of wheel and rail wear under different contact conditions using artificial neural networks," Wear, vol. 406-407, pp. 173-184, 2018.

[13] E. Butini et al., "An innovative model for the prediction of wheel - Rail wear and rolling contact fatigue," Wear, vol. 436-437, 2019.

[14] R. Lewis, W. J. Wang, M. Burstow, and S. R. Lewis, "Investigation of the influence of rail hardness on the wear of rail and wheel materials under dry conditions," Civil-Comp Proc., vol. 110, 2016.

[15] C. Chang, B. Chen, Y. Cai, and J. Wang, "An experimental study of high speed wheel-rail adhesion characteristics in wet condition on full scale roller rig," Wear, vol. 440-441, 2019.

[16] Z. Wei, A. Núñez, X. Liu, R. Dollevoet, and Z. Li, "Multicriteria evaluation of wheel/rail degradation at railway crossings," Tribol. Int., vol. 144, 2020.

[17] L. Ling, M. Dhanasekar, and D. P. Thambiratnam, "A passive road-rail crossing design to minimise wheel-rail contact failure risk under frontal collision of trains onto stuck trucks," Eng. Fail. Anal., vol. 80, pp. 403-415, 2017.
[18] S. Morales-Ivorra, J. I. Real, C. Hernández, and L. Montalbán, "Derailment risk and dynamics of railway vehicles in curved tracks: Analysis of the effect of failed fasteners," J. Mod. Transp., vol. 24, no. 1, pp. 38-47, 2016.

[19] J. Xu, Y. Gao, P. Wang, B. An, J. Chen, and R. Chen, "Numerical analysis for investigating wheel-rail impact contact in a flange bearing frog crossing," Wear, vol. 450 451, 2020.

[20] D. Thomas, B. Diedrichs, M. Berg, and S. Stichel, "Dynamics of a high-speed rail vehicle negotiating curves at unsteady crosswind," Proc. Inst. Mech. Eng. Part F J. Rail Rapid Transit, vol. 224, no. 6, pp. 567-579, 2010.

[21] L. H. Wang, A. N. Huang, and G. W. Liu, "Analysis on curve negotiation ability of the rail vehicle based on SIMPACK," Adv. Mater. Res., vol. 721, pp. 551-555, 2013.

[22] X. Xiao, X. Jin, Y. Deng, and Z. Zhou, "Effect of curved track support failure on vehicle derailment," Veh. Syst. Dyn., vol. 46, no. 11, pp. 1029-1059, 2008.

[23] K. Wang, C. Huang, W. Zhai, P. Liu, and S. Wang, "Progress on wheel-rail dynamic performance of railway curve negotiation," J. Traffic Transp. Eng. (English Ed., vol. 1, no. 3, pp. 209-220, 2014. 